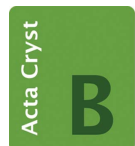

STRUCTURAL SCIENCE CRYSTAL ENGINEERING MATERIALS

ISSN 2052-5206

Received 23 October 2018

Accepted 7 December 2018

Keywords: phase transition; polymorphism; polytypism; twinning; order-disorder (OD) theory.

CCDC references: 1883688; 1883689; 1883690; 1883691; 1883692; 1883693; 1883694

Supporting information: this article has supporting information at journals.iucr.org/b

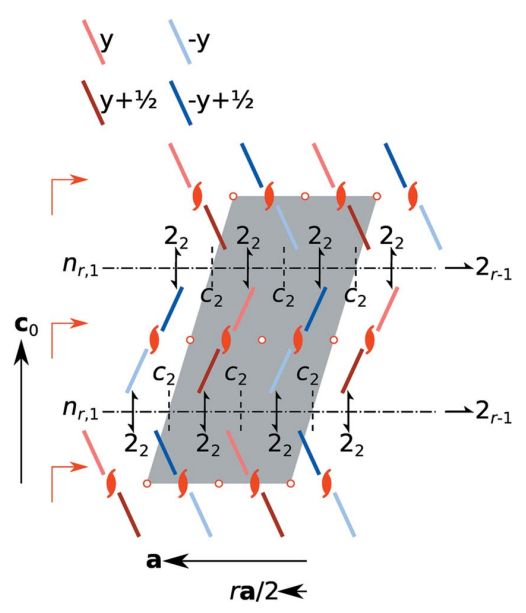

OPEN $\odot$ ACCESS

\section{The phase transitions of 4-aminopyridine-based indolocarbazoles: twinning, local- and pseudo- symmetry}

\author{
Thomas Kader, ${ }^{a}$ Berthold Stöger, ${ }^{b *}$ Johannes Fröhlich $^{\mathrm{a}}$ and Paul Kautny ${ }^{\mathrm{a}}$ \\ ${ }^{\mathbf{a}}$ Institute of Applied Synthetic Chemistry, TU Wien, Getreidemarkt 9, 1060 Vienna, Austria, and ${ }^{\mathbf{b} X-R a y ~ C e n t r e, ~ T U ~ W i e n, ~}$ \\ Getreidemarkt 9, 1060 Vienna, Austria. ${ }^{*}$ Correspondence e-mail: bstoeger@mail.tuwien.ac.at
}

The phase transitions and polymorphism of three 4-aminopyridine-based indolocarbazole analogues are analyzed with respect to symmetry relationships and twinning. Seven polymorphs were structurally characterized using singlecrystal diffraction. $5 \mathrm{NICz}$ (the indolo[3,2,1-jk]carbazole derivative with the $\mathrm{C}$ atom in the 5-position replaced by $\mathrm{N}$ ) crystallizes as a $P 2_{1} / a$ high-temperature $(270 \mathrm{~K})$ polymorph and as a $P c a 2_{1}$ low-temperature $(150 \mathrm{~K})$ polymorph. Even though their space-group symmetry is not related by a group-subgroup relationship, the local symmetries of both belong to the same order-disorder (OD) groupoid family. Both are polytypes of a maximum degree of order and are twinned by point operations of the other polytype. $2 \mathrm{NICz}(\mathrm{C}$ atom in the 2position replaced by $\mathrm{N}$ ) likewise crystallizes in a high-temperature (Pcca, $280 \mathrm{~K}$ ) polymorph and a low-temperature $\left(P 2_{1} / c, 150 \mathrm{~K}\right)$ polymorph. Here, the spacegroup symmetries are related by a group-subgroup relationship. The lowtemperature phase is twinned by the point operations lost on cooling. The crystal structure of bulk $2,5 \mathrm{NICz}$ (N-substitution at the 2- and 5-positions) was unrelated to $2 \mathrm{NICz}$ and $5 \mathrm{NICz}$ and no phase transition was observed. Isolated single crystals of a different polymorph of $2,5 \mathrm{NICz}$, isotypic with $2 \mathrm{NICz}$, were isolated. However, the analogous phase transition in this case takes place at distinctly higher temperatures $(>300 \mathrm{~K})$.

\section{Introduction}

Symmetry relationships are crucial in understanding and describing phase transitions (Müller, 2013). In most cases of displacive phase transition (Tolédanoc et al., 2006), the symmetry of a high-temperature (HT) phase is a strict super group of the symmetry of the low-temperature (LT) phase (disregarding minor variations of cell parameters). Nevertheless, exceptions exist. For example, numerous incommensurate phases feature a lock-in phase transition to a periodic (and therefore higher-symmetry) LT structure on cooling (Cummins, 1990). In such a case, both phases are derived from a higher-symmetry prototype structure, which may exist at high temperatures or may be purely hypothetical.

Reconstructive phase transitions are generally not restricted by group-subgroup relationships because, as the name implies, a significant rearrangement of atoms or molecules takes place. There are intermediate cases of symmetry transformations, where modules (layers, rods) are preserved but are arranged differently. In such a case, an interpretation using local symmetry can be insightful.

In this context, we present the structural phase transitions of three 4-aminopyridine derivatives of indolo[3,2,1-jk]carbazole $(\mathrm{ICz})$, whereby $\mathrm{C}$ atoms para to the $\mathrm{N}$ atom of $\mathrm{ICz}$ are replaced by an $\mathrm{N}$ atom. The IUPAC atom numberingscheme is given in Fig. 1(a). The molecules under investiga- 
tion, $5 \mathrm{NICz}, 2 \mathrm{NICz}$ and $2,5 \mathrm{NICz}$ feature substitution of $\mathrm{C}$ atoms by $\mathrm{N}$ at the respective positions [Figs. $1(b)-1(d)$ ].

Crystals of $5 \mathrm{NICz}$ and $2 \mathrm{NICz}$ exist in distinct $\mathrm{HT}$ and LT polymorphs, which interconvert below room temperature. $2,5 \mathrm{NICz}$ exists in the solid state as three polymorphs. The bulk $2,5 \mathrm{NICz}-1$ crystallizes in a structure unrelated to $5 \mathrm{NICz}$ and $2 \mathrm{NICz}$. We could not find any evidence of a phase transition in the solid state for this polymorph. While attempting to obtain improved diffraction data, we found isolated crystals of a different polymorph, which is isostructural to $2 \mathrm{NICz}$. These crystals featured an analogous phase transition (polymorphs designated 2,5NICz-2LT and 2,5NICz-2HT), though with a transition temperature above room temperature.

The observed phase transitions are analyzed with respect to symmetry relationships. Whereas the symmetries of $2 \mathrm{NICz}-\mathrm{LT}$ and 2NICz-HT polymorphs (and the 2,5NICz-2LT and $2,5 \mathrm{NICz}-2 \mathrm{HT}$ polymorphs) can be described using classical group-subgroup relationships, the local symmetry has to be considered for $5 \mathrm{NICz}$. For this purpose, we use the formalism developed in the framework of order-disorder (OD) theory (Dornberger-Schiff \& Grell-Niemann, 1961; Ferraris et al., 2008). Despite being of the same name, this theory of polytypism is not related to order-disorder phase transitions. A summary of the phase transitions and the structural relationships between the seven polymorphs is schematized in Fig. 2.

\section{Experimental}

\subsection{Synthesis and crystal growth}

The molecules under investigation were synthesized by ring closure of 9-(2-bromophenyl)-9H-carbazole derivatives with the appropriate $\mathrm{N}$-substitution patterns using $5 \mathrm{~mol} \%$ of an allyl[1,3-bis(2,6-diisopropylphenyl)imidazol-2-ylidene]chloropalladium(II) catalyst. Reaction optimization studies and full

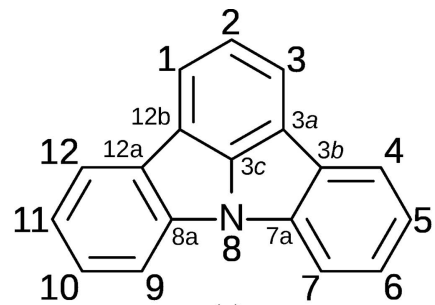

(a)

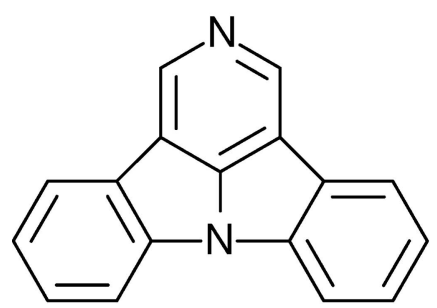

(c)

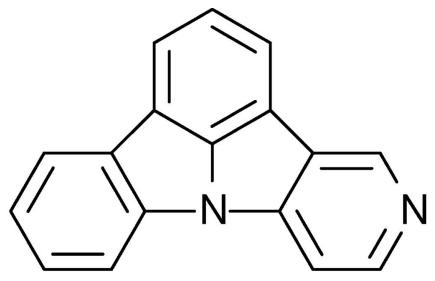

(b)

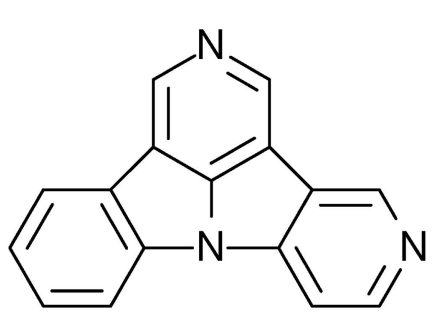

(d)
Figure 1

Schematics of (a) ICz with IUPAC numbering scheme and $(b-d)$ the 4aminopyridine derivatives $5 \mathrm{NICz}, 2 \mathrm{NICz}$ and $2,5 \mathrm{NICz}$. characterizations are given by Kader et al. (2019). Crystals were grown by slow evaporation of acetonitrile solutions.

To prepare $5 \mathrm{NICz}$, a glass vial was charged with 9-(3bromopyridin-4-yl)-9H-carbazole (1 equiv., $324 \mathrm{mg}, 1 \mathrm{mmol}$ ), $\mathrm{K}_{2} \mathrm{CO}_{3}$ (2 equiv., $276 \mathrm{mg}, 2 \mathrm{mmol}$ ) and $\mathrm{Pd}$ catalyst ( 0.05 equiv., $29 \mathrm{mg}, 0.05 \mathrm{mmol}$ ) and flushed with argon. After addition of $10 \mathrm{ml}$ degassed $N, N$-dimethylacetamide, the reaction was stirred under argon atmosphere until full conversion. The cooled reaction mixture was poured into water and extracted into $\mathrm{CH}_{2} \mathrm{Cl}_{2}$. The organic phase was dried over $\mathrm{Na}_{2} \mathrm{SO}_{4}$ and concentrated under reduced pressure. The crude product was purified by column chromatography. $2 \mathrm{NICz}$ was prepared according to the same procedure starting from 5-(2-bromophenyl)-5H-pyrido[4,3-b]indole (1 equiv. $322 \mathrm{mg}, 1 \mathrm{mmol}$ ). Column chromatography afforded fractions of pure $2 \mathrm{NICz}$ as well as mixtures of $2 \mathrm{NICz}$ and $5 \mathrm{NICz}$. 2,5NICz was prepared according to the same procedure starting from 5-(3-bromopyridin-4-yl)-5H-pyrido[4,3- $b$ ]indole (1 equiv., $324 \mathrm{mg}$, $1 \mathrm{mmol}) .5,11 \mathrm{NICz}$ was obtained as secondary product.

\subsection{Data collection and refinement}

2.2.1. General. Intensity data were collected in a dry stream of nitrogen on a Bruker Kappa APEX II diffractometer system using graphite-monochromated Mo $K \bar{\alpha}$ radiation. Data were reduced to intensity values using SAINT (Bruker, 2017). Corrections for absorption and related effects were applied using $S A D A B S$ (Bruker, 2017). The structures were solved with SHELXT (Sheldrick, 2015a) and refined with SHELXL (Sheldrick, 2015b). The atoms were labelled according to IUPAC rules (Fig. 1a). In the case of two crystallographically different molecules $\left(Z^{\prime}=2\right)$, prime characters are added for the second molecule. For molecules located on twofold axes, atoms pairs that are equivalent by symmetry are assigned the lower out of the two possible numbers. More data collection

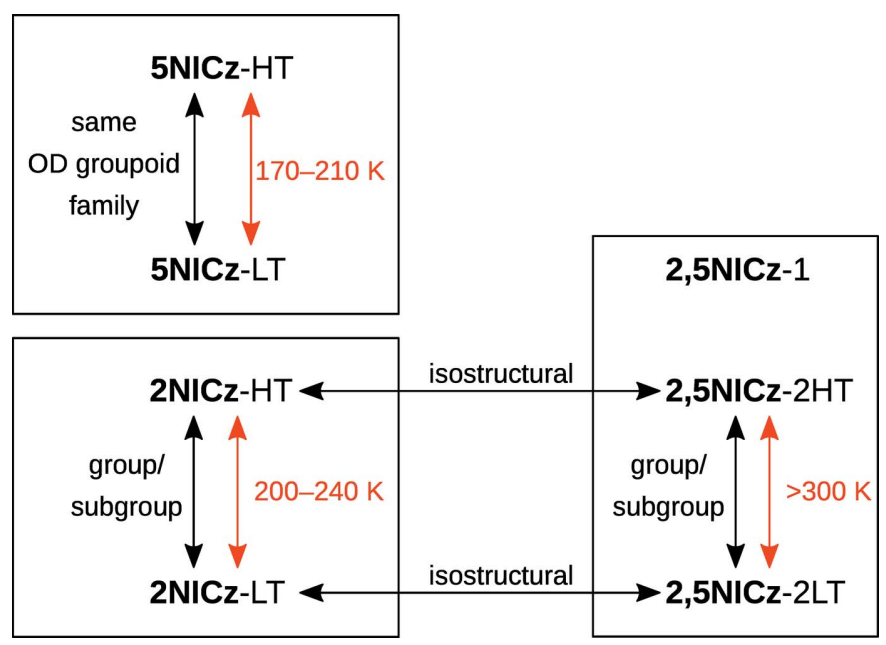

Figure 2

Phase transition temperatures (red arrows) and structural relationships (black arrows) relating the seven polymorphs described in this work. The meaning of 'same OD groupoid family' is explained at the end of \$3.1.1. Polymorphs not connected by arrows are structurally unrelated. 
Table 1

Experimental details.

For all structures: colourless crystals, Mo K $\alpha$ radiation, Bruker Kappa APEX II CCD diffractometer, multi-scan absorption correction, H-atom parameters constrained.

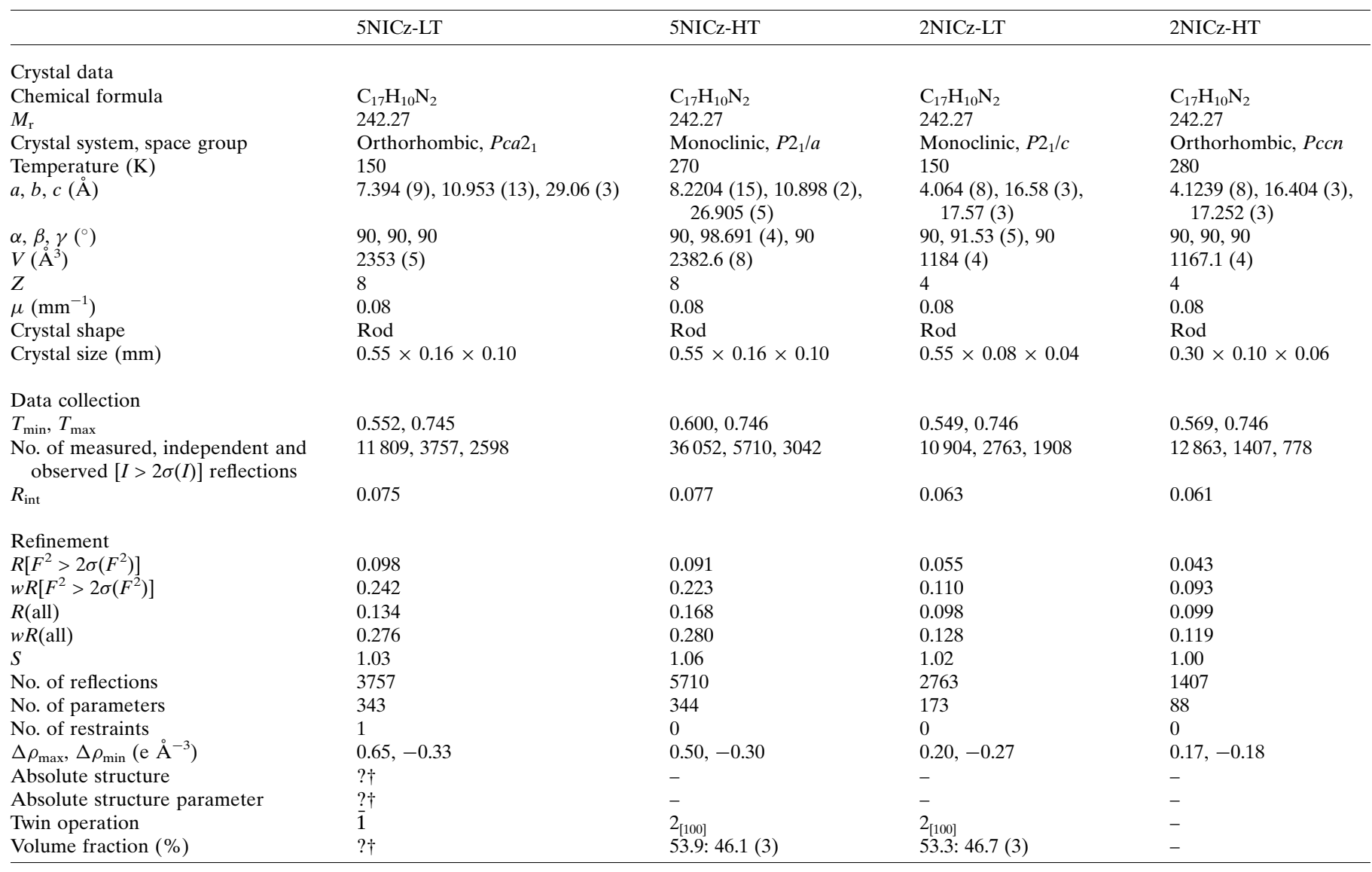

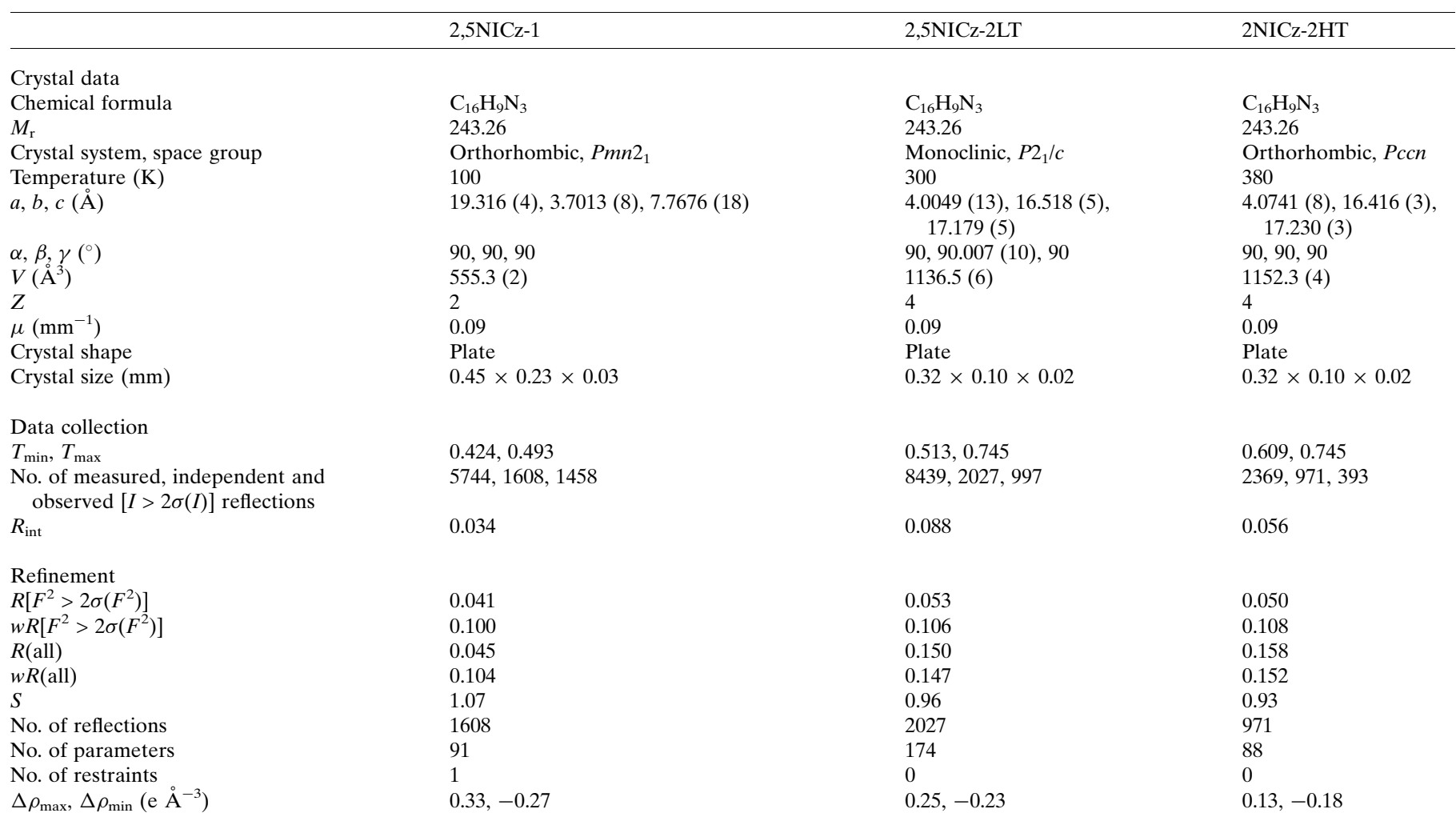


Table 1 (continued)

\begin{tabular}{|c|c|c|c|}
\hline & $2,5 \mathrm{NICz}-1$ & 2,5NICz-2LT & $2 \mathrm{NICz}-2 \mathrm{HT}$ \\
\hline Absolute structure & $\begin{array}{l}\text { Flack } x \text { determined using } 561 \\
\quad \text { quotients }[(\mathrm{I}+)-(\mathrm{I}-)] /[(\mathrm{I}+)+(\mathrm{I}-)] \\
\quad \text { (Parsons } \text { et al., } 2013)\end{array}$ & - & - \\
\hline Absolute structure parameter & $-0.3(10)$ & - & - \\
\hline Twin operation & $? \dagger$ & $2_{[100]}$ & - \\
\hline Volume fraction (\%) & $? \dagger$ & $54.7: 45.3(4)$ & - \\
\hline
\end{tabular}

Computer programs: SAINT, APEXII, SADABS (Bruker, 2017), SHELXL2014/7 (Sheldrick, 2015b). $\dagger$ Not determined owing to a lack of significant resonant scatterers.

and refinement details are summarized in Tables 1 and 2, and described in the following sections.

2.2.2. Details for 5 NICz. Crystals of 5 NICz were small, yet of reasonable quality according to optical microscopy. Nevertheless, in preliminary scans at the routine temperature of $150 \mathrm{~K}$, all plates featured mediocre reflection quality and diffracted only to small $2 \theta$ angles. Such a bad diffraction quality for optically flawless crystals can be a sign of a reconstructive phase transition on cooling. Indeed, crystals cooled to $150 \mathrm{~K}$ showed clear signs of fracturing. Two data sets were, therefore, collected with long exposure times, one above the phase transition temperature at $270 \mathrm{~K}$ and one after slow cooling to $150 \mathrm{~K}$. To our surprise, even at $270 \mathrm{~K}$ the reflection quality was not significantly improved.

For the $150 \mathrm{~K}$ data set a reasonable structure solution and refinement, considering the mediocre diffraction quality, was possible in the space group $P c a 2_{1}$.

The $270 \mathrm{~K}$ phase had apparent orthorhombic $C$-centred $(o C)$ metrics. But, since a sensible structure solution was not possible in this setting and slight splitting of reflections indicated a lower metric symmetry, the data were reprocessed in the corresponding monoclinic primitive $(m P)$ setting. Structure solutions and refinements were performed in the space group $P 2_{1} / a$ under consideration of twinning by pseudomerohedry. The non-standard setting of the space group $P 2_{1} / c$ was chosen to ease comparison with the LT polymorph.

2.2.3. Details for $2 \mathrm{NICz}, 2,5 \mathrm{NICz}-2 \mathrm{LT}$ and 2,5NICz-2HT. The cell parameters of the LT-polymorph of $2 \mathrm{NICz}$ were apparently orthorhombic primitive $(o P)$ and, therefore, data were at first processed assuming such a symmetry. A structure solution was successful in the space group Pccn. But all attempts at refinements resulted in excessively anisotropic atomic displacement parameters (ADPs) and mediocre residuals. Since, in analogy to $5 \mathrm{NICz}$, reflections at higher diffraction angles were split, an attempt was made in the $P 2_{1} / c$ space group under consideration of twinning by pseudomerohedry. The ADPs as well as the residuals improved significantly $\left(R_{\mathrm{obs}}>10 \%\right.$ to $\sim 5.5 \%$ ). For the HT phase, on the other hand, a refinement using Pccn symmetry was successful. In this case, reducing the symmetry to monoclinic did not improve reliability factors.

The cell parameters of $2,5 \mathrm{NICz}-2 \mathrm{~L} \mathrm{~T}$ suggested a structure isostructural to 2NICz. Refinements were, therefore, performed using starting models derived from the $2 \mathrm{NICz}$ model. Even at $300 \mathrm{~K}$, refinements in the LT $P 2_{1} / c$ model resulted in significantly improved residuals $\left(R_{\mathrm{obs}}>10 \%\right.$ to $\sim 5.3 \%$ ), even though the metrics are orthorhombic within the estimated standard errors. Only when heating to $380 \mathrm{~K}$ was the Pccn HT phase clearly observed. In 2,5NICz-2HT, the molecules are located on a twofold axis and the $\mathrm{N} 5$ atom is accordingly positionally disordered with the $\mathrm{C} 11$ atom in a 1:1 manner. In 2,5NICz-2LT, this position splits in two and both positions were refined as positionally disordered, by constraining the sum of the $\mathrm{N}$-occupancies of both positions to 1. Ultimately, the $\mathrm{N}$-occupancy of one position refined 0.58 (4) (the other accordingly being constrained to 0.42 ).

2.2.4. Details for $2,5 \mathrm{NICz}-1$. The structure of $2,5 \mathrm{NICz}-1$ was determined by routine refinement. The $2,5 \mathrm{NICz}$ molecule is located on a twofold axis and, therefore, the N5 and C11 atoms are positionally disordered in a 1:1 manner.

\subsection{X-ray powder diffraction}

Low-temperature X-ray powder diffraction (XRPD) experiments were performed on a Panalytical X'Pert Pro diffractometer equipped with an Oxford Cryosystems PheniX closed cycle cryostat in Bragg-Brentano geometry using $\mathrm{Cu} K \alpha_{1,2}$ radiation $(\lambda=1.540598,1.544426 \AA)$ with an Ni filter and an X'celerator multi-channel detector. The ground bulk sample was placed on an Si single crystal cut along the (711) plane. Scans were recorded in vacuum in the $2 \theta=10-70^{\circ}$ range in $10 \mathrm{~K}$ steps from $300 \mathrm{~K}$ to $100 \mathrm{~K}$ and back to $300 \mathrm{~K}$ with heating and cooling rates of $1 \mathrm{~K} \mathrm{~min}^{-1}$ and $5 \mathrm{~min}$ isothermals between scans.

\section{Results and discussion \\ 3.1. The OD polytypism of $5 \mathrm{NICz}$}

3.1.1. Local symmetry. The HT and LT phases of $5 \mathrm{NICz}$ are structurally closely related. They crystallize in the $P 12_{1} / a 1$ and $P c a 2_{1}$ symmetry, respectively and contain $Z^{\prime}=25 \mathrm{NICz}$ molecules in the asymmetric unit. The structures can be considered as being composed of $A_{n}$ layers ( $n$ is a sequential integer) extending parallel to (001) (Fig. 3). These layers are made up of rods of molecules which connect via short $\mathrm{C}-$ $\mathrm{H} \cdots \mathrm{N}$ contacts (Fig. 4). Whereas these rods are very similar in both structures (differences will be discussed below), their inclination with respect to the layer plane (001) differs significantly (Fig. 3). The angles of the least-squares planes of the molecules to the (001) plane are $67.5^{\circ}$ and $67.6^{\circ}$ versus $55.7^{\circ}$ and $56.5^{\circ}$ for the LT and HT phases, respectively. Thus, the two kinds of layers can be derived from each other, but they might not be considered as isostructural (Kálmán et al., 1993) in the strict sense. 
Adjacent molecules in the rods described above are related by a $2_{1}$ operation in the [010] direction. The operation is exact in the HT phase (one crystallographically unique molecule per rod) but only approximate in the LT phase (two molecules per rod). Adjacent rods are, in both polymorphs, related by an $a$ operation in the [010] direction. Moreover, they are related by inversions, which is a space group operation in the HT and a local operation in the LT phase. Thus, the layers possess $P 12_{1} / a(1)$ actual (HT) or pseudo (LT) symmetry. Since we will perform an interpretation according to the OD theory, here we use the OD notation of layer symmetry, whereby parentheses indicate the direction lacking translational symmetry (Dornberger-Schiff \& Grell-Niemann, 1961). In the LT polymorph, adjacent $A_{n}$ layers are related by actual $2_{1}$ screw rotations in [001] and $c$ glide reflections in [100] direction, whereas in the HT polymorph, these are only a pseudo-

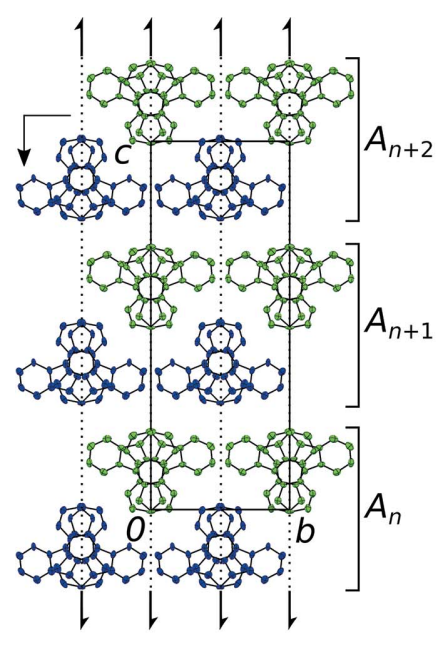

(a)

(b)
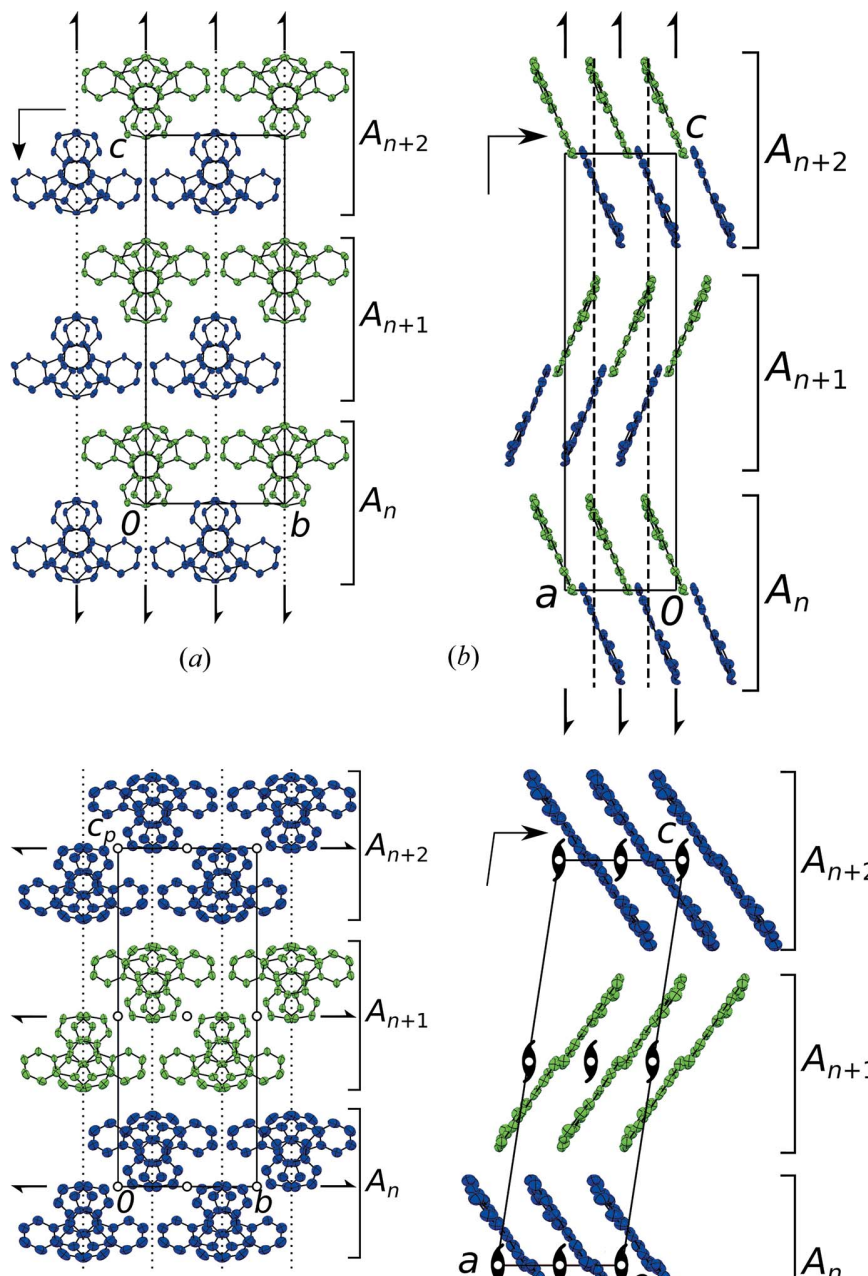

(c)

(d)

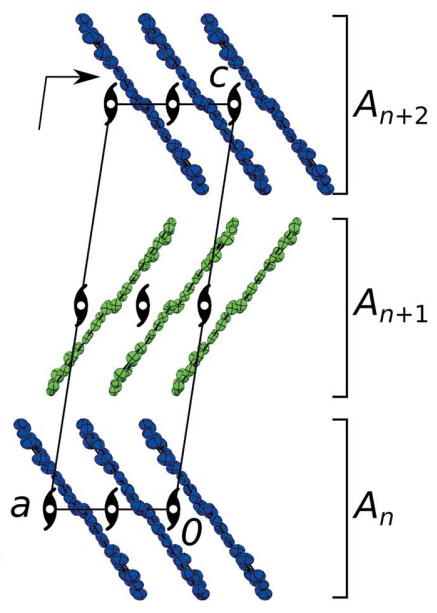

Figure 3

The $(a, b)$ LT and $(c, d)$ HT polymorphs of $5 \mathrm{NICz}$ viewed down $(a, c)$ [100] and $(b, d)$ [010]. Molecules are coloured according to space-group symmetry equivalence. $\mathrm{H}$ atoms are omitted for clarity. Layer names are indicated to the right. Crystallographic symmetry elements are indicated by the common graphical symbols (Hahn \& Aroyo, 2016). symmetry operations. In total, both polymorphs are made up of $Z^{\prime}=2$ crystallographically different molecules.

Recognizing the pseudo-symmetry of layers is the key to an OD interpretation. By assuming the pseudo-symmetry to be exact, both polymorphs can be described as members of OD families. The symmetries of OD families are classified into OD groupoid families, which correspond to space group types in

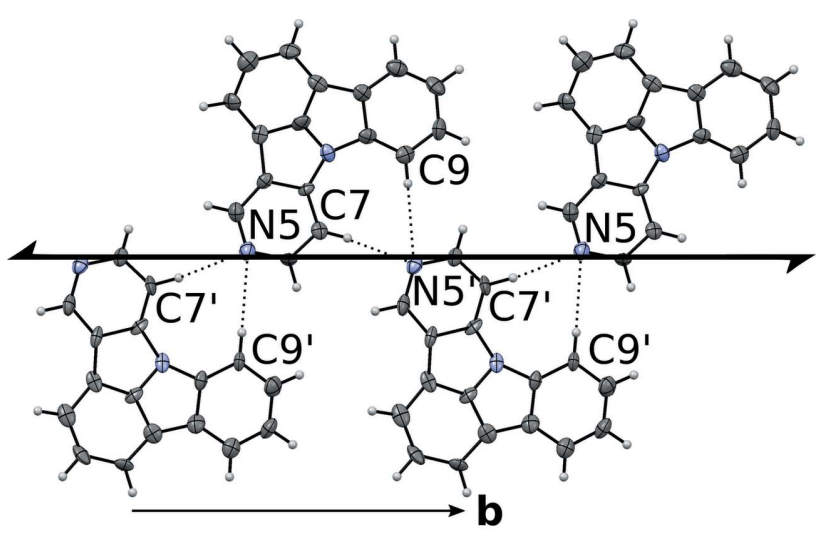

(a)

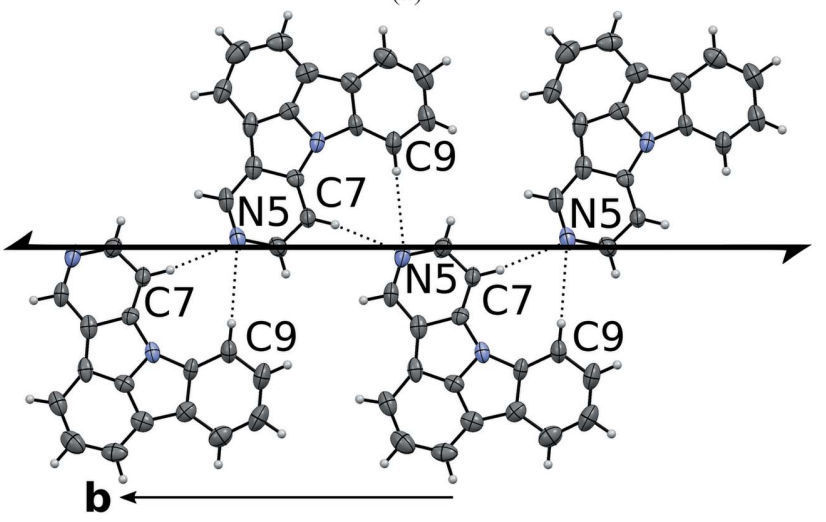

(b)

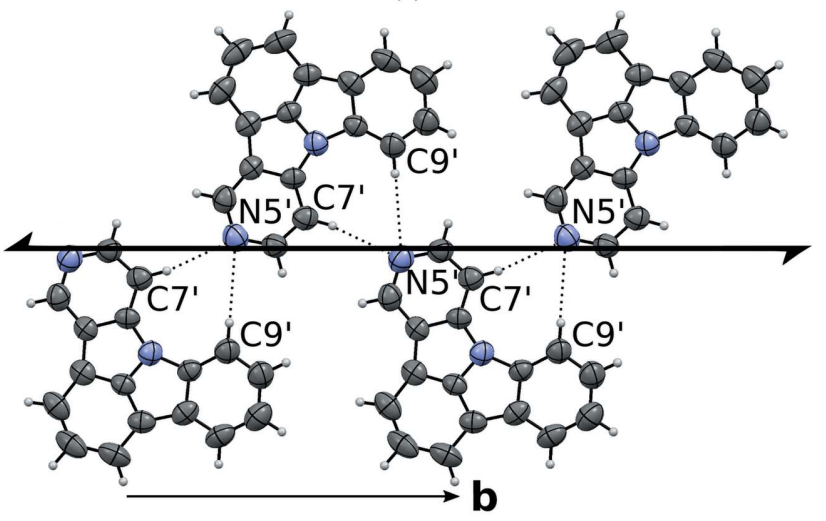

(c)

Figure 4

Rods of $5 \mathrm{NICz}$ molecules connected by $\mathrm{C}-\mathrm{H} \cdots \mathrm{N}$ interactions (dotted lines) extending along [010] in the $(a)$ LT and $(b, c)$ HT polymorphs. Note that in the HT polymorph there are two kinds of rods, whereas in the LT polymorph all rods are related by symmetry. $\mathrm{C}$ and $\mathrm{N}$ atoms are represented by grey and blue ellipsoids, respectively, drawn at the $50 \%$ probability levels, $\mathrm{H}$ atoms by white spheres of arbitrary radius. Pseudo (a) and crystallographic $(b, c) 2_{1}$ screw axes are indicated using the usual symbol (Hahn \& Aroyo, 2016). 
classical crystallography (Fichtner, 1979a). The symmetries of both polymorphs belong to the same OD groupoid family, which is described by

$$
\left.\begin{array}{cccc}
P & 1 & 21 / a & (1) \\
\{ & 2_{r-1} / n_{s-1,2} & - & \left(2_{2} / n_{r, s}\right)
\end{array}\right\}
$$

according to the notation of Dornberger-Schiff \& GrellNiemann (1961). The metric parameter $s$ adopts the value $s=1$ in both cases, which can be expressed by

$$
\left.\begin{array}{cccc}
P & 1 & 21 / a & (1) \\
\{ & 2_{r-1} / c_{2} & - & \left(2_{2} / n_{r, 1}\right)
\end{array}\right\} .
$$

OD groupoids are made up of partial operations (POs), which relate layers but need not apply to the whole stacking sequence. The first line in these symbols gives the symmetry group of the layers [the $\lambda$-POs, here $\left.P 12_{1} / a(1)\right]$. The second line lists one possible set of operations relating adjacent layers ( $\sigma$-POs). Since the relative intrinsic translations of the $\sigma$-POs are not restricted to those found in space groups, generalized Hermann-Mauguin symbols are used. For example, the $n_{r, s}$ glide reflection in the symbol above has the glide vector $r \mathbf{a} / 2+s \mathbf{b} / 2$. As can be seen in Fig. 3, the $x$-component of the glide vector is approximately $\frac{1}{4}$ and thus $r \approx \frac{1}{2}(\$ 3.1 .5)$.

Intrinsic translation components in the stacking direction [001] are given with respect to the vector $\mathbf{c}_{0}$, which is perpendicular to the layers and of the length of one layer width. Thus, the $2_{2}$ operation in [001] direction has the screw vector $\mathbf{c}_{0}$ (Fichtner, 1979b) since $n_{m}$ stands for an $n$-fold screw rotation with intrinsic translation of $\frac{m}{n}$ parts of the shortest lattice vector in the translation direction.

3.1.2. Stacking possibilities. The crucial aspect of OD structures is their ability of crystallizing in different polytypes, which are all locally equivalent (more precisely: pairs of adjacent layers are equivalent). If interactions beyond one layer width and deviations from the prototype layers are neglected, all polytypes can therefore be considered as energetically equivalent. The $N F Z$ relationship (Ďurovič, 1997) is used to derive these stacking possibilities. For $5 \mathrm{NICz}$, there are $\sigma$-POs that invert the orientation with respect to the stacking direction ( $\sigma$ - $\rho$-POs). But owing to $r \notin \mathbb{Z}$ none of these is a reverse continuation, which would mean that it maps $A_{n}$ on $A_{n+1}$ and vice versa. In such a case, the NFZ relationship reads as $Z=2 N / F=2\left[\mathcal{G}_{n}: \mathcal{G}_{n} \cap \mathcal{G}_{n+1}\right]$, where $Z$ is the number of positions $A_{n+1}$ can adopt given $A_{n}$ and $\mathcal{G}_{n}$ is the group of those $A_{n}$ operations that do not invert the orientation with respect to the stacking direction $(\lambda-\tau$-POs).

Since $s=1$, the $a$ glide planes of all $A_{n}$ overlap and $\mathcal{G}_{n}=\mathcal{G}_{n} \cap \mathcal{G}_{n+1}=P 1 a(1)$. Accordingly, there are $Z=2[P 1 a(1): P 1 a(1)]=2$ ways of placing $A_{n+1}$ given $A_{n}$. These two possibilities are obtained by applying a $2_{r-1}$ or a $2_{1-r} \sigma$-PO on $A_{n}$, respectively.

3.1.3. Maximum degree of order (MDO) polytypes. Out of the infinity of stacking arrangements, two are of a MDO, which means that they cannot be decomposed into fragments of simpler polytypes (Dornberger-Schiff, 1982). $\mathrm{MDO}_{1}\left[P 2_{1} / a\right.$, $\left.\mathbf{c}=(r-1) \mathbf{a}+2 \mathbf{c}_{0}\right]$ is generated by repeated application of $2_{r-1} \sigma$-POs; $\mathrm{MDO}_{2}\left[\mathrm{Pca}_{1}, \mathbf{c}=2 \mathbf{c}_{0}\right]$ by alternating application of $2_{r-1}$ and $2_{1-r} \sigma$-POs. The local symmetry of both polytypes is schematized in Fig. 5.

In our experience, the overwhelming number of polytypes characterized by single-crystal diffraction is of the MDO kind. Other stacking arrangements may exist at domain interfaces. Indeed, the HT and LT polymorphs of $5 \mathrm{NICz}$ are precisely of the $\mathrm{MDO}_{1}$ and $\mathrm{MDO}_{2}$ type, respectively. Thus, even though the space groups of the two phases are not related by a groupsubgroup relationship, their groupoids belong to the same groupoid family with the same restrictions on the metric parameters, viz. $s=1$. Their local symmetries are therefore, in a sense, isomorphic, which demonstrates the usefulness of such a symmetry description.

3.1.4. Twinning. Crystals of OD polytypes are often twinned owing to stacking faults. The possible orientation states of the polytype are derived by coset decomposition of the point group of the polytype in the point group of the OD groupoid family, that is the point group generated by the

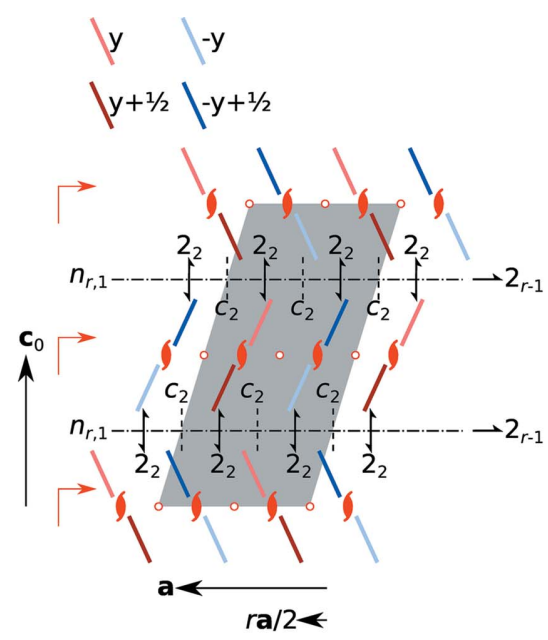

(a)

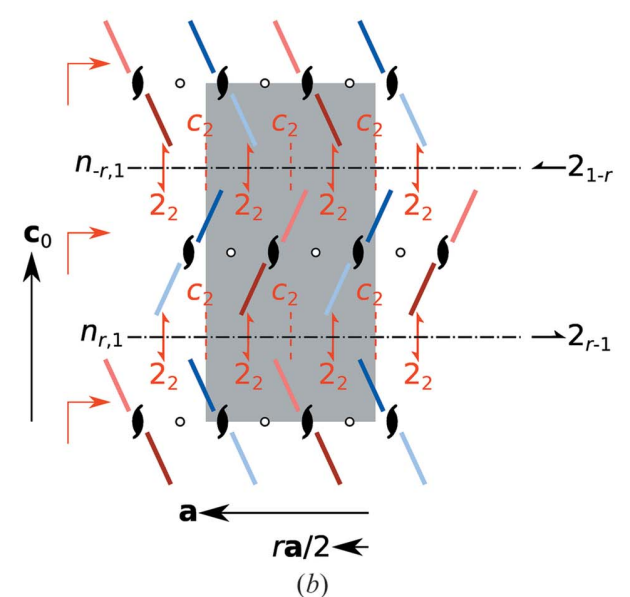

Figure 5

Symmetry of the $\mathrm{MDO}_{1}$ and $\mathrm{MDO}_{2}$ polytypes of $5 \mathrm{NICz}$ viewed along $\mathbf{b}$. Molecules are represented by bars which are red on one side and blue on the other. Darker colours indicate translation by $\mathbf{b} / 2$. $\lambda$-POs of the layers and $\sigma$-POs relating adjacent layers are indicated by the common graphical symbols (Hahn \& Aroyo, 2016) and, in the case of unusual intrinsic translations, by their printed symbols. Symbols of POs that are valid for the whole polytype are red. The unit cell of the polytypes is marked by a grey backdrop. 
linear parts of all POs of a polytype. This group is $m m m$ for the OD groupoid family of the $5 \mathrm{NICz}$ polymorphs.

Thus, $\mathrm{MDO}_{1}(\mathrm{HT})$ can appear in $[\mathrm{mmm}: 12 / \mathrm{m} 1]=2$ orientations, which are related by the operations of the twin law $\left\{2_{x}, m_{x}, 2_{z}, m_{z}\right\}$. This corresponds precisely to the observed twinning. $\mathrm{MDO}_{2}$ (LT) can appear likewise in [ $\left.\mathrm{mmm}: \mathrm{mm} 2\right]=2$ orientations. In this case, the twin law is $\left\{\overline{1}, 2_{x}, 2_{y}, m_{z}\right\}$. Since the $5 \mathrm{NICz}$ molecules possess no significant resonant scatterers under Mo $K \alpha$ radiation, this twinning by inversion could not be seen from the diffraction data. Its existence is nevertheless nearly certain. Besides being predicted by OD theory, it is also expected owing to the phase transition from the centrosymmetric $\mathrm{MDO}_{1}(\mathrm{HT})$ phase. Point operations lost on phase transformation are typically retained as twin operations.

3.1.5. Desymmetrization and metric parameters. An important step in assessing an OD model is the quantification of the desymmetrization (Durovič, 1979) compared to the ideal model. Such a desymmetrization is expected (these geometrical differences may stabilize the individual polytypes) but should not be unreasonably large.

In the $\mathrm{MDO}_{1}(\mathrm{HT})$ polytype, the symmetry of the actual $A_{n}$ layers is identical to those of the idealized description $\left[P 12_{1} / a(1)\right]$. According to the $P 2_{1} / a$ symmetry of the polytypes, the layers are partitioned into two equivalence classes, viz. the $A_{2 n}$ and the $A_{2 n+1}$ layers. To evaluate the desymmetrization, the $A_{1}$ layer was mapped onto the $A_{0}$ layer by translation of $-\mathbf{c} / 2$ and reflection at the $\mathbf{r} \cdot \mathbf{a}=0$ plane. The discrepancies between both layers are minute (max: $\mathrm{C} 2 / \mathrm{C}^{\prime}$, $0.157 \AA$ ), proving the validity of the pseudo-symmetry analysis.

In the $\mathrm{MDO}_{2}$ (LT) polytype all layers are related by the $P c a 2_{1}$ space group symmetry, but the symmetry of the layers is reduced by an index of 2 to $P 1 a(1)$. To assess the degree of desymmetrization, the location of the pseudo- $2_{1}$ screw axis was determined by averaging the $x$ - and $z$-coordinates of the non- $\mathrm{H}$ atoms of the two crystallographically independent molecules. The screw rotation was then applied to a layer. Here, the desymmetrization is even less pronounced than in the HT phase (max: $\mathrm{C} 11 / \mathrm{C} 11^{\prime}, 0.086 \AA$ ).

The metric parameter $r$ of the OD groupoids can be derived in the case of $\mathrm{MDO}_{1}(\mathrm{HT})$ directly from the cell parameters as $r=c \cos \beta / a+1=0.505$. Owing to $r \approx 1 / 2$, the lattice symmetry of $\mathrm{MDO}_{1}$ is pseudo-oC and the twinning is by pseudo-merohedry (the reflections of both domains are nearly coincident). More precisely, the twin obliquity calculates from the cell parameters as $\omega=\tan ^{-1}\left[a\left(r-\frac{1}{2}\right) / c / \sin \beta\right] \approx 0.1^{\circ}$. It has to be noted though that the derivation of the cell parameters from single-crystal data is inexact in such a case because overlapping reflections are treated as single reflections during integration. The deviation from $r=1 / 2 \mathrm{might}$, therefore, be larger than estimated here.

For $\mathrm{MDO}_{2}(\mathrm{LT}), r$ is derived from the $x$-coordinate of the pseudo- $2_{1}$ operation (see above) as $r=4 x=0.449$. Thus, in both cases, despite the distinctly different orientation of the molecules, the parameter $r$ is approximately $1 / 2$.

3.1.6. Structural changes on phase transition. Even though symmetry considerations are the main focus of this work,
Table 2

Non-classical $\mathrm{C}-\mathrm{H} \cdots \mathrm{N}$ hydrogen bonding in both polymorphs of $5 \mathrm{NICz}$.

\begin{tabular}{lllll}
\hline$D-\mathrm{H} \cdots A$ & $\begin{array}{l}D-\mathrm{H} \\
(\AA)\end{array}$ & $\begin{array}{l}\mathrm{H} \cdots A \\
(\AA)\end{array}$ & $\begin{array}{l}D \cdots A \\
(\AA)\end{array}$ & $\begin{array}{l}D-\mathrm{H} \cdots A \\
\left({ }^{\circ}\right)\end{array}$ \\
\hline
\end{tabular}

$150 \mathrm{~K}$

$\mathrm{C} 7-\mathrm{H} \cdots \mathrm{N} 5^{\prime}$

$\mathrm{C} 9-\mathrm{H} \cdots \mathrm{N} 5^{\prime}$

$\mathrm{C}^{\prime}-\mathrm{H} \cdots \mathrm{N} 5$

$\mathrm{C} 9^{\prime}-\mathrm{H} \cdots \mathrm{N} 5$

$270 \mathrm{~K}$

$\mathrm{C} 7-\mathrm{H} \cdots \mathrm{N} 5$

$\mathrm{C} 9-\mathrm{H} \cdots \mathrm{N} 5$

$\mathrm{C}^{\prime}-\mathrm{H} \cdots \mathrm{N} 5^{\prime}$

$\mathrm{C} 9^{\prime}-\mathrm{H} \cdots \mathrm{N} 5^{\prime}$

0.95
0.95
0.95
0.95

0.93
0.93
0.93
0.93

$3.389(14)$

162.7

$0.95 \quad 2.80$

$0.95 \quad 2.51$

$\begin{array}{ll}0.95 & 2.78\end{array}$

$3.722(14)$

$3.426(14)$

163.2

160.7

$3.706(15)$

163.8

0.93

0.93

0.93

0.93

2.53

2.86

3.446 (9)

$3.784(9)$

$3.404(5)$

167.0

173.8

165.0

2.87

$3.789(7)$

172.1

changes at the crystallo-chemical level must not be neglected. As has been noted above, the structures of both $5 \mathrm{NICz}$ polymorphs are controlled by non-classical $\mathrm{C}-\mathrm{H} \cdots \mathrm{N}$ hydrogen interactions, forming chains extending in the [010] direction (Fig. 4). Each molecule forms a pocket delimited by $\mathrm{N} 8$ and the $\mathrm{H} 7$ and $\mathrm{H} 9$ are in meta position to N8. These two $\mathrm{H}$ atoms are expected to be the most 'acidic' and indeed interact with the N5 lone pair of the adjacent molecule. The hydrogen

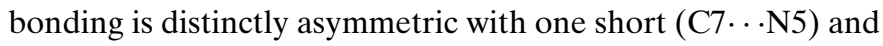

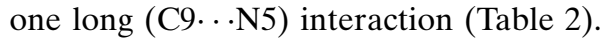

The $\mathrm{C} \cdots \mathrm{N}$ distances are slightly longer in the HT phase. In return, the $\mathrm{C}-\mathrm{H} \cdots \mathrm{N}$ angles are closer to linear, owing to near coplanarity of the connected molecules [Fig. 3(d)]. Overall, the hydrogen bonding can be considered as close to equivalent in both polymorphs.

Adjacent rods are connected by $\pi-\pi$ interactions to layers. Here, the structural changes on phase transition are significant owing to a change in molecule inclination with respect to the

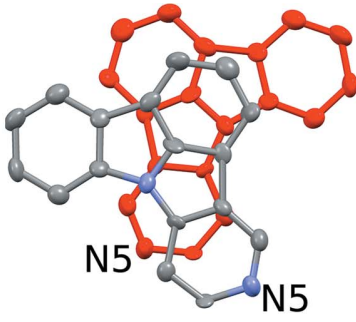

(a)

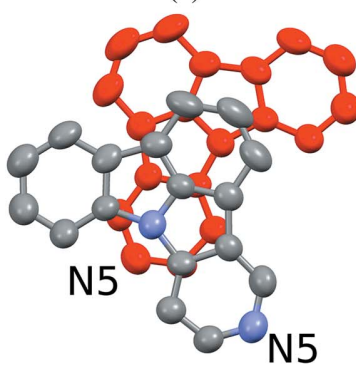

(c)

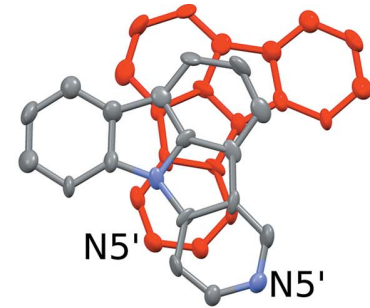

(b)

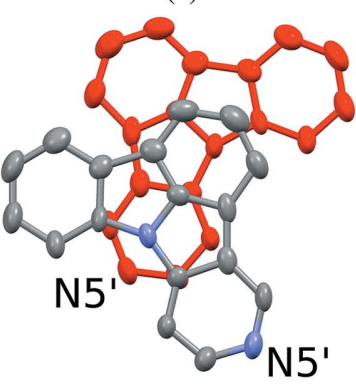

$(d)$
Figure 6

Pairs of $5 \mathrm{NICz}$ molecules connected by $\pi-\pi$ interactions in the $(a, b)$ LT and $(c, d)$ HT polymorphs, projected on the molecular plane. Atom colours of the top molecules as in Fig. 4; bottom molecules in red for clarity. Ellipsoids are drawn at the $50 \%$ probability levels. 
layer plane (Fig. 6). The $\mathrm{C}-\mathrm{H} \cdots \pi$ contacts relating adjacent layers are, like the hydrogen bonding, very similar in both polymorphs. In summary, the dominant factor in the phase transition seems to be the $\pi-\pi$ stacking.

\subsection{Phase transitions of $2 \mathrm{NICz}$, and $2,5 \mathrm{NICz}-2 \mathrm{LT}$ and 2,5NICz-2HT polymorphs}

3.2.1. Symmetry relationships. The $2 \mathrm{LT}$ and $2 \mathrm{HT}$ polymorphs of $2,5 \mathrm{NICz}$ are isostructural to the corresponding LT and $\mathrm{HT} 2 \mathrm{NICz}$ polymorphs, whereby the N5 and C11 atoms are positionally disordered. In contrast to $5 \mathrm{NICz}$, the symmetries of the respective HT and LT polymorphs are related by a group-subgroup relationship. As is often observed in such a case, the symmetry of the HT phase $\left(P 2_{1} / c 2_{1} / c 2 / n, Z=4\right)$ is a strict super group (here minimal) of the symmetry of the LT phase $\left(P 12_{1} / c 1, Z=4\right)$.

The structures are again built up of rods of $2 \mathrm{NICz}$ $(2,5 \mathrm{NICz})$ molecules connected by short $\mathrm{C}-\mathrm{H} \cdots \mathrm{N}$ interactions extending along [001] (Fig. 7). In the HT phase, the molecules are located on a twofold rotation axis and adjacent molecules are related by $c_{[100]}$ and $c_{[010]}$ glide reflections. The rods, therefore, possess $p c c 2$ symmetry (Kopsky \& Litvin, 2006). In the [100] direction, adjacent rods are generated by lattice translations. From a thus constructed layer, the final

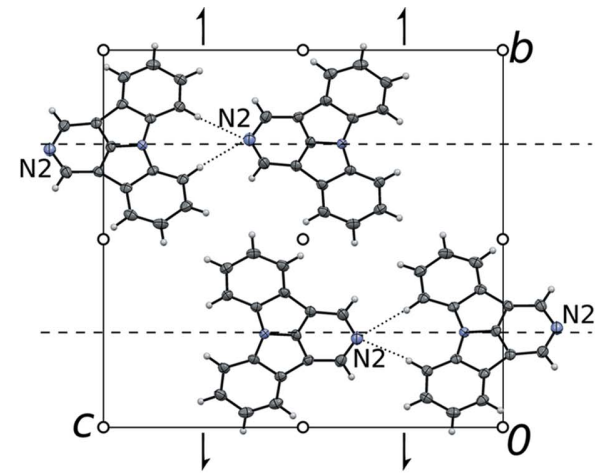

(a)

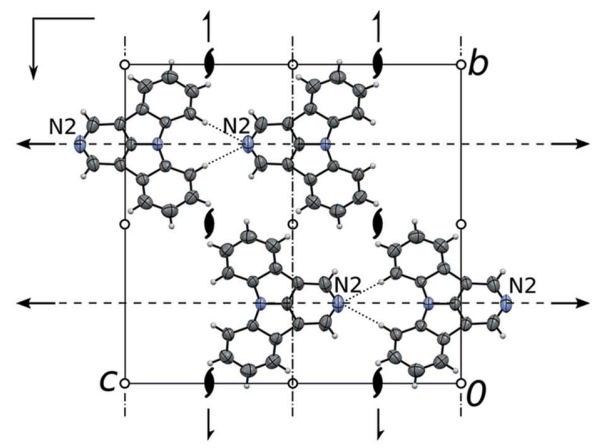

(c)

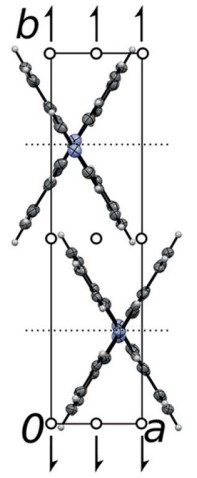

(b)

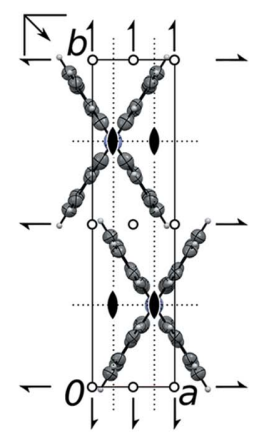

(d)
Figure 7

Crystal structures of the $(a, b)$ LT and $(c, d)$ HT polymorphs of $2 \mathrm{NICz}$ viewed down $(a, c)$ [100] and $(b, d)[001]$. Atom colours as in Fig. 4. The 2LT and $2 \mathrm{HT}$ polymorphs of $2,5 \mathrm{NICz}$ are isostructural and not shown. Ellipsoids are drawn at the $50 \%$ probability levels. Crystallographic symmetry elements are indicated by the common graphical symbols (Hahn \& Aroyo, 2016). structure with Pccn symmetry is generated by $2_{1}$ screw rotations in the [010] direction.

In the LT phase, the twofold rotation symmetry of the rods is lost. Of the two $c$-glide reflections, only the operation with plane parallel to (010) is retained. Thus, the symmetry of the rods is reduced by an index of 2 from $\mu c c 2$ to $p 1 c 1$. The rods are again related by translations forming layers parallel to (010) and the whole structure then generated by $2_{1}$ screw rotations in [010] direction, resulting in an overall $P 2_{1} / c$ symmetry.

3.2.2. Twinning. Whereas the HT polymorphs are not twinned, on cooling below the phase transition temperature, the lost point operations are retained as twin operations. The twin law is obtained as a coset of the coset decomposition of the LT in the HT point group. Thus, the LT twin consists of $[\mathrm{mmm}: 2 / \mathrm{m}]=2$ domains, whose orientations are related by the operations $\left\{2_{x}, m_{x}, 2_{z}, m_{z}\right\}$. The twinning is by pseudo-merohedry, since the orthorhombic metrics of the lattice are approximately retained. The twin obliquity is derived from the cell parameters as $1.5^{\circ}$ (2NICz-LT) and $0.0^{\circ}(2,5 \mathrm{NICz}-2 \mathrm{LT})$. Indeed, for $2,5 \mathrm{NICz}-2 \mathrm{LT}$ no splitting of reflections was observed in single-crystal experiments, whereas for $2 \mathrm{NICz}-\mathrm{LT}$ the twin obliquity is reflected in rows of diverging reflections.

3.2.3. Desymmetrization. The deviation of $\beta$ from ideal orthorhombic metrics is a measure of desymmetrization. For a finer evaluation of the desymmetrization, the atomic coordinates were transformed in an orthonormal coordinate system and the pseudo-rotation axis located at $\left(\frac{1}{4}, \frac{1}{4}, z\right)$ was applied to a molecule. The atoms in the original and the transformed molecule are separated by $0.52-0.68 \AA$ (2NICz-LT) and $0.08-$ $0.53 \AA(2,5 \mathrm{NICz}-2 \mathrm{LT})$. Whereas in $2 \mathrm{NICz}-\mathrm{LT}$ the deviation is mostly due to a translation away from the rotation axis, in

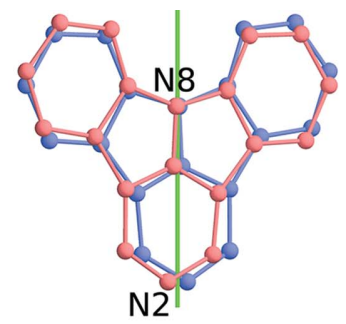

(a)

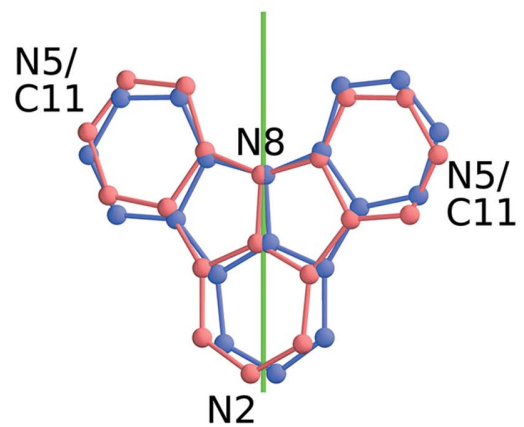

(c)

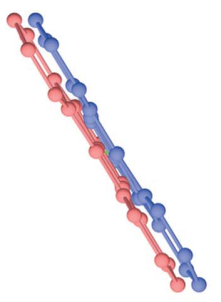

(b)

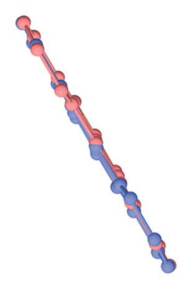

(d)
Figure 8

Overlay of molecules and their images by twofold rotation about the $\left(\frac{1}{4}, \frac{1}{4}, z\right)$ axis in the $(a, b) 2 \mathrm{NICz}-\mathrm{LT}$ and $(c, d) 2,5 \mathrm{NICz}-2 \mathrm{LT}$ polymorphs viewed down $(a, c)$ [100] and $(b, d)$ [001]. The rotation axis is shown in green. 
Table 3

Non-classical $\mathrm{C}-\mathrm{H} \cdots \mathrm{N}$ hydrogen bonding in the $\mathrm{HT}$ and $\mathrm{LT}$ polymorphs of $2 \mathrm{NICz}$ and the $2 \mathrm{HT}$ and $2 \mathrm{LT}$ polymorphs of $2,5 \mathrm{NICz}$.

\begin{tabular}{|c|c|c|c|c|}
\hline$D-\mathrm{H} \cdots A$ & $\begin{array}{l}D-\mathrm{H} \\
(\AA)\end{array}$ & $\begin{array}{l}\mathrm{H} \cdots A \\
(\AA)\end{array}$ & $\begin{array}{l}D \cdots A \\
(\AA)\end{array}$ & $\begin{array}{l}D-\mathrm{H} \cdots A \\
\left(^{\circ}\right)\end{array}$ \\
\hline \multicolumn{5}{|l|}{ 2NICz-LT } \\
\hline $\mathrm{C} 7-\mathrm{H} 7 \cdots \mathrm{N} 2$ & 0.95 & 2.59 & $3.540(6)$ & 174.5 \\
\hline $\mathrm{C} 9-\mathrm{H} 9 \cdots \mathrm{N} 2$ & 0.95 & 2.65 & $3.594(6)$ & 174.7 \\
\hline 2NICz-HT & & & & \\
\hline $\begin{array}{l}\mathrm{C} 7-\mathrm{H} 7 \cdots \mathrm{N} 2(2 \times) \\
25 \mathrm{NIC7}-2 \mathrm{LT}\end{array}$ & 0.93 & 2.60 & $3.532(2)$ & 175.6 \\
\hline $\begin{array}{l}2,5 \mathrm{NICz}-2 \mathrm{LT} \\
\mathrm{C} 7-\mathrm{H} 7 \cdots \mathrm{N} 2\end{array}$ & 0.93 & 2.58 & $3.505(6)$ & 177.7 \\
\hline $\mathrm{C} 9-\mathrm{H} 9 \cdots \mathrm{N} 2$ & 0.93 & 2.60 & $3.529(6)$ & 175.5 \\
\hline 2,5NICz-2HT & & & & \\
\hline $\mathrm{C} 7-\mathrm{H} 7 \cdots \mathrm{N} 2(2 \times)$ & 0.93 & 2.61 & $3.538(5)$ & 175.7 \\
\hline
\end{tabular}

2,5NICz-2LT the molecules are tilted with respect to the rotation axis of the HT phase (Fig. 8).

3.2.4. Crystal chemistry. As in the case of $5 \mathrm{NICz}$, the central crystallo-chemical feature are rods connected by nonclassical $\mathrm{C}-\mathrm{H} \cdots \mathrm{N}$ hydrogen bonding involving the two $\mathrm{H} 7$ and $\mathrm{H} 9$ positions. Here, the bonding is more symmetrical, with two equivalent (HT) or only slightly different (by ca $0.05 \AA$; LT) bonds (Table 3). Enlarged ADPs of the N2 atom (Fig. 7c) indicate that the desymmetrization is dynamic, i.e. the orientations of the molecules oscillate between the two possible asymmetric states. Since the remaining structural changes are likewise minute, one can assume that the desymmetrization of the hydrogen-bonding is the decisive factor in the phase transition. Numerous reported solid-solid phase transitions

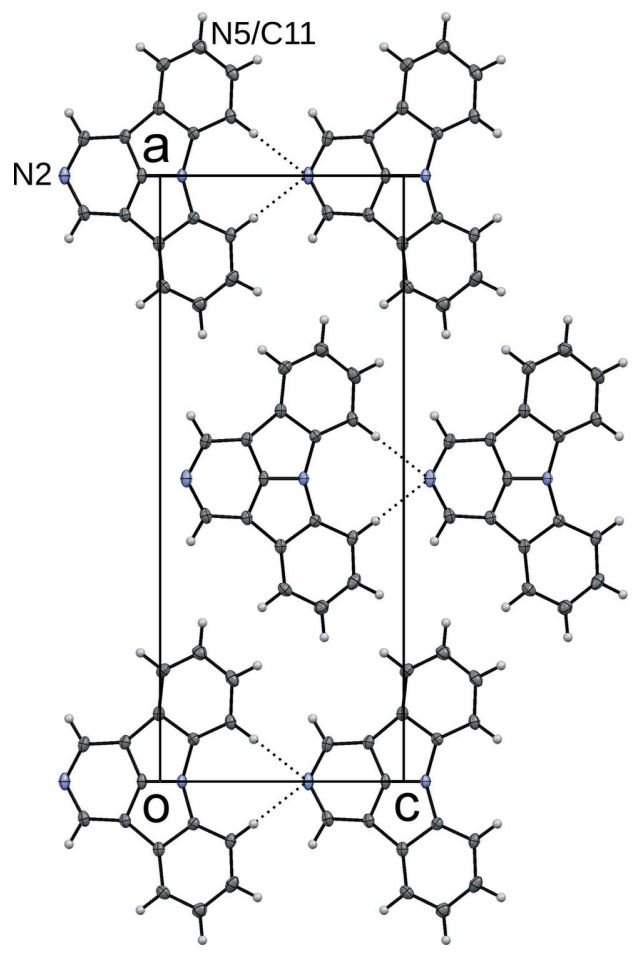

Figure 9

The crystal structure of 2,5NICz-1 viewed down [010]. Atom colours as in Fig. 4. are due to such a dynamic desymmetrization, a classical example being the $\mathrm{KH}_{2} \mathrm{PO}_{4}$ (KDP) family of ferroelectrics (Peercy, 1975).

\section{3. $2,5 \mathrm{NICz}-1$}

The bulk polymorph 2,5NICz-1 features a crystallographically non-challenging structure with $P m n 2_{1}$ symmetry. In analogy to the other structures presented here, the $2,5 \mathrm{NICz}$ molecules are connected by hydrogen bonds to chains (Fig. 9). In contrast to the $2,5 \mathrm{NICz}-2 \mathrm{LT}$ and $2,5 \mathrm{NICz}-2 \mathrm{HT}$ polymorphs, the connected molecules are coplanar (related by a $\mathbf{b}+\mathbf{c}$ lattice translation), demonstrating that the inclination is determined by packing effects.

\subsection{Powder diffraction}

To determine the stability ranges of the LT and HT polymorphs and to rule out additional phase transitions, powdered samples of $5 \mathrm{NICz}$ and $2 \mathrm{NICz}$ were subjected to lowtemperature powder diffraction (Fig. 10). In a bulk sample of $2,5 \mathrm{NICz}$ only the orthorhombic polymorph 1 could be seen by $\mathrm{X}$-ray diffraction, which does not possess a phase transition in the solid state. Thus, in this case the exact phase transition temperature could not be determined. In both cases, $5 \mathrm{NICz}$ and $2 \mathrm{NICz}$, the $\mathrm{HT} \leftrightarrow \mathrm{LT}$ transitions are clearly showed by appearance/vanishing of peaks and a distinct hysteresis of $\sim 20 \mathrm{~K}$ is observed [5NICz: transitions at $180-170 \mathrm{~K}$ (cooling) versus 200-210 K (heating); 2NICz: 210-200 K (cooling) versus $230-240 \mathrm{~K}$ (heating)]. No other phase transitions are apparent. The hysteresis suggests a phase transition of the first order. Even though neither powder diffraction nor DSC data for the 2,5NICz-2 polymorph could be acquired, experiments on the single crystal showed a smooth transition to the orthorhombic phase. This phase transition might be, therefore, of the second order.

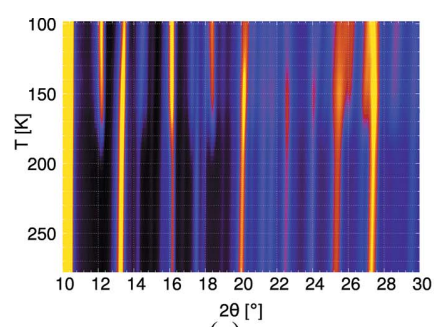

(a)

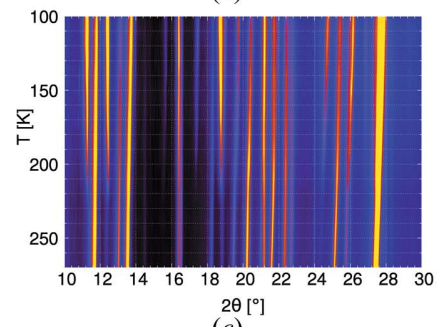

(c)

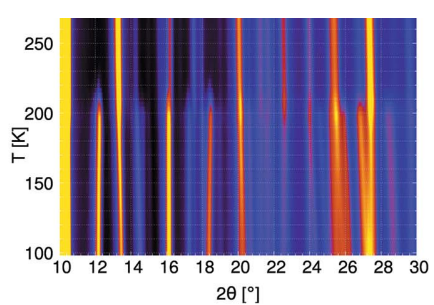

(b)

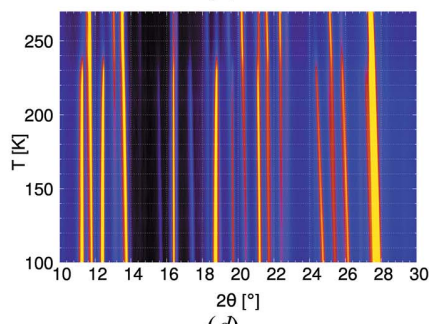

(d)
Figure 10

Low-temperature XRPD scans of $5 \mathrm{NICz}(a, b)$ and $2 \mathrm{NICz}(c, d)$ over the $2 \theta$ range $10-30^{\circ}$ on heating $(a, c)$ and cooling $(b, d)$ shown as heat maps. Maximum and minimum intensities are yellow and black, respectively. 


\section{Conclusion}

From a crystallo-chemical point of view, the polymorphs of $5 \mathrm{NICz}, 2 \mathrm{NICz}$ and $2,5 \mathrm{NICz}$ are all closely related. Their structures are determined by non-classical $\mathrm{C}-\mathrm{H} \cdots \mathrm{N}$ hydrogen bonding. The molecular orientations in the thusformed rods differ owing to either $\mathrm{N}$-substitution at different rings or with respect to the rotation of adjacent molecules.

Nevertheless, they are fundamentally different from a crystallographical point of view. The transitions between 2NICz-LT and 2NICz-HT, and the isostructural $2,5 \mathrm{NICz}-2 \mathrm{LT}$ and $2,5 \mathrm{NICz}-2 \mathrm{HT}$ are clearly displacive and, as expected in such a case, the symmetries of the polymorphs are related by a group-subgroup relationship. The transition of $5 \mathrm{NICz}$, on the other hand, is a borderline case between displacive and reconstructive, with layers that are in principle similar but feature distinctly changed inclination of the molecules. More interestingly, the symmetry relationship between both polymorphs can only be understood by analysis of their space groupoids in the sense of OD theory. Thus, it is shown that a unified theory of local symmetry is needed.

\section{Acknowledgements}

The authors thank Werner Artner for performing the lowtemperature powder X-ray diffraction experiments.

\section{Funding information}

TK, JF and PK gratefully acknowledge financial support by the Austrian Science Fund (FWF) (grant No. I 2589-N34).

\section{References}

Bruker (2017). APEXII, SAINT and SADABS. Bruker Analytical $\mathrm{X}$-ray Instruments, Inc., Madison, WI, USA.

Cummins, H. Z. (1990). Phys. Rep. 185, 211-409.

Dornberger-Schiff, K. (1982). Acta Cryst. A38, 483-491.

Dornberger-Schiff, K. \& Grell-Niemann, H. (1961). Acta Cryst. 14, 167-177.

Ďurovič, S. (1979). Krist. Techn. 14, 1047-1053.

Ďurovič, S. (1997). EMU Notes Mineral. 1, 3-28.

Ferraris, G., Makovicky, E. \& Merlino, S. (2008). Crystallography of Modular Materials, vol. 15 of IUCr Monographs on Crystallography. Oxford University Press.

Fichtner, K. (1979a). Krist. Techn. 14, 1073-1078.

Fichtner, K. (1979b). Krist. Techn. 14, 1453-1461.

Hahn, Th. \& Aroyo, M. I. (2016). Symbols of symmetry elements, International Tables For Crystallography, Vol. A, Space-group symmetry, 1st online ed., ch. 2.1.2, pp. 144-148. Chester: International Union of Crystallography.

Kader, T., Stöger, B., Fröhlich, J. \& Kautny, P. (2019). Chem. Eur. J. In the press, doi: 10.1002/chem.201805578.

Kálmán, A., Párkányi, L. \& Argay, G. (1993). Acta Cryst. B49, 10391049.

Kopský, V. \& Litvin, D. B. (2006). Editors. International Tables for Crystallography, Vol. E, Subperiodic groups, 1st online ed. Chester: International Union of Crystallography.

Müller, U. (2013). Symmetry Relationships between Crystal Structures. Oxford University Press.

Parsons, S, Flack, H. D. \& Wagner, T. (2013). Acta Cryst. B69, 249259.

Peercy, P. S. (1975). Phys. Rev. B, 12, 2725-2740.

Sheldrick, G. M. (2015a). Acta Cryst. A71, 3-8.

Sheldrick, G. M. (2015b). Acta Cryst. C71, 3-8.

Tolédano, J., Janovec, V. V., Kopský, V., Scott, J. F. \& Boček, P. (2006). International Tables for Crystallography, Vol. D, Physical properties of crystals, edited by A. Authier, pp. 338-376. Chester, England: International Union of Crystallography. 